\title{
EFFECT OF CIRCUIT TRAINING IN OSTEOARTHRITIS OF KNEE
}

\author{
PALLAVI BHAGAT**, VAISHALI JAGTAP, POOVISHNU DEVI T
}

Department of Musculoskeletal Sciences, Krishna College of Physiotherapy, KIMSDU, Karad - 415 110, Maharashtra, India. Email: bhagat15pallavi@gmail.com

Received: 07 March 2017, Revised and Accepted: 19 April 2017

\section{ABSTRACT}

Objectives: The objective of the study is to find the effect of circuit training on quality of life. To find the effect of conventional therapy (interferential therapy [IFT] and isometric exercise) and to find the effect of conventional therapy with or without circuit training in OA knee.

Methods: A total of 30 participants of 50-60 years of age, having osteoarthritis (OA) knee were recruited and allocated into two groups and treated with IFT, exercises, and circuit training for 4 weeks. Pre- and post-intervention outcome were measured using Western Ontario and McMaster University OA Index (WOMAC) and visual analog scale (VAS).

Result: Both groups showed improvement, but there was an extremely significant improvement on VAS and WOMAC scales in the group treated with circuit training along with conventional treatment (IFT and isometric exercises)

Conclusion: From this study, we conclude that circuit training along with IFT and isometric exercises shows an extremely significant effect over IFT and isometric exercises alone in OA knee patients.

Keywords: Osteoarthritis, Interferential therapy, Western Ontario and McMaster University Osteoarthritis Index, Visual analog scale, Circuit training.

(C) 2017 The Authors. Published by Innovare Academic Sciences Pvt Ltd. This is an open access article under the CC BY license (http://creativecommons. org/licenses/by/4. 0/) DOI: http://dx.doi.org/10.22159/ajpcr.2017.v10i7.18322

\section{INTRODUCTION}

Osteoarthritis $(\mathrm{OA})$ is a non-inflammatory degenerative disorder of joints characterized by progressive deterioration of articular cartilage and formation of new bone (osteophytes) [1]. Knee OA is a prevalent condition that contributes significantly to functional limitations and disability in older people. Physical impairments associated with knee OA include pain, loss of motion, and decreased muscle strength $[2,3]$. The individual of any age can be affected with arthritis; the usual age of onset is between 25 and 50 with a peak in the 40 and 50 seconds [4]

The prevalence of OA increases with age in both sexes, the Knee Joint in women is particularly susceptible to this insidious disease. OA is most frequent joint disease with the prevalence of 22-39\% in India [5]. There are many reasons for the high prevalence of $\mathrm{OA}$ in India like popular squatting position in India, rising obesity, sedentary lifestyle, and poor diet are responsible for its high incidence [6].

\section{LITERATURE REVIEW}

Gabriela Hernandez-Molina, MD, MSc, Stephan Reichenbach, MD, Bin Zhang, PhD, Michael LaValley, PhD, and David T Felson MD, MPH. Effect of therapeutic exercise for hip OA pain: Results of a meta-analysis. This study shows the therapeutic exercise, especially with an element of strengthening, is an efficacious treatment for hip 0A [7].

Sao Paulo Med J Vol. 129(4), Sao Paulo 2011. Effect on transcutaneous nerve stimulation (TENS) and interferential therapy (IFT) in a patient with nonspecific chronic low back pain: Randomized clinical trial. The results from this study showed that TENS and IFC had significant effects in relation to pain intensity reduction, disability improvement, and reduction of medication consumption, immediately after each electrotherapy session and after ten sessions, in comparison with the controls. However, no significant differences in these resources in relation to treating patients with nonspecific chronic low back pain were observed [8].

\section{METHOD}

Totally, 30 participants with $\mathrm{OA}$ knee willing to take treatment for 4 weeks were recruited for the study. The subjects were screened and were put in either of the groups Group A (circuit training, IFT, and exercises) and Group B (IFT and exercises) by simple random sampling with randomized allocation method. A written informed consent was taken from each participant. Ethical Clearance was obtained from University's Institutional Review Board. Inclusion criteria were both male and female subjects between 50 and 60 years of age symptomatically and radiologically diagnosed with Stage I and II OA knee fulfilling Altman and Colleague criteria for OA of the knee joint.

Exclusion criteria were other rheumatologic conditions, recent fracture around the knee, open wound or skin disease, secondary OA, lower limb deformities, osteoporosis, bone tumors, and previous knee surgeries.

Both groups were treated with IFT with the frequency of $4000 \mathrm{~Hz}$. For the duration of 10 minutes for 4 weeks [9]. The exercise program included static quadriceps, static hamstrings exercises, and circuit training includes warm up for 5 minutes, repetition of walking, straight lunges, one leg balance, balance training using wobble board, for 20 minutes/session, for 4 weeks, 3 times/weeks, 3 sets.

\section{Outcome measure}

Subjects in both groups were evaluated pre- and post-treatment program using Western Ontario and McMaster Universities OA Index (WOMAC) scale and visual analog scale (VAS).

\section{WOMAC scale}

The WOMAC is used to assess patients with OA of knee using 24 items [10].

The VAS is used to assess pain in patients with OA of knee

\section{Statistical analysis}

Statistical analysis for the present study was done manually as well as using the instruction statistics so as to verify the results obtained. 
Various statistical measures such as paired t-test, unpaired t-test were used for this purpose.

Intragroup comparison (within the group) was analyzed statistically using paired t-test for WOMAC and VAS score, intergroup comparison (between group) was analyzed statistically using an unpaired t-test for WOMAC and VAS $p<0.05$ were considered statistically significant and $\mathrm{p}<0.0001$ were considered statistically extremely significant.

\section{RESULTS}

A total of 30 participants were taken for study. The gender ratio of Group A was 8:7 (8 males and 7 females) and Group B was 10:5 (10 males and 5 females) and was statistically not significant. Therefore, both groups are matched with respect to gender.

The age of the participants in the study was between 50 and 60 years. The mean age of the participants in Group A was 57.44 \pm 2.354 years, and the mean age of participants in Group B was $55.4 \pm 3.50$ years. The difference in mean age of two groups was statistically not significant $(p=0.1104)$. Therefore, both groups are matched with respect to age (Table 1).

The table also shows the comparison of mean and standard deviation (SD) of pre- and post-values of Group A and B. In the Group A, the mean VAS score on pre-intervention was $6.22 \pm 2.100$ which was reduced to a mean of $1.933 \pm 0.8837$ post sessions. The $p$ value by paired $t$-test was found to be $<0.0001$ which is extremely significant.

In Group B, the mean VAS score on pre-intervention was $5.5 \pm 1.669$ which was reduced to a mean of $3.8 \pm 1.612$ post-interventions. The $p$ value by paired $t$-test found to be $<0.0001$ which is extremely significant (Table 2 ).

The table also shows the comparison of mean and SD of pre- and post-values of Group A and B. In the Group A, the mean VAS score on pre-intervention was $6.22 \pm 2.100$ which was reduced to a mean of $1.933 \pm 0.8837$ post sessions. The $p$ value by paired $t$-test was found to be $<0.0001$ which is extremely significant.

In Group B, the mean VAS score on pre-intervention was $5.5 \pm 1.669$ which was reduced to a mean of $3.8 \pm 1.612$ post-interventions. The $\mathrm{p}$ value by paired t-test found to be $<0.0001$ which is extremely significant (Table 3).

Table 1: Baseline characteristics of participants

\begin{tabular}{lll}
\hline Variable & Group A & Group B \\
\hline Sex & Male=8 and Female $=7$ & Male $=10$ and Female $=5$ \\
Age (years) & $57.4 \pm 2.354$ & $55.4 \pm 3.501$ \\
Side affected & $\mathrm{Rt}=8$ and $\mathrm{Lt}=9$ & $\mathrm{Rt}=7$ and $\mathrm{Lt}=6$ \\
\hline
\end{tabular}

On comparing the pre-interventional values, the results between the two groups using unpaired t-test revealed that there was no statistically significant difference seen with $\mathrm{p}=0.3075$. While on comparing the postsession values, the results between the two groups using unpaired t-test revealed that there was extremely significant difference seen with $\mathrm{p}=3.932$.

The table shows a comparison of mean values and SD of VAS scores in Group A and Group B. The values were compared by applying unpaired t-test. Pre-treatment shows that there is no significant difference in the VAS scores $(p=0.3075)$, whereas posttreatment shows extremely significant difference $(\mathrm{p}=3.932)$ (Table 4).

On comparing the precession values, the results between the two groups using unpaired t-test revealed that there was no statistically significant difference seen with $p$ values of 0.5515 . While on comparing the post-interventional values, the results between the two groups using unpaired t-test revealed that there was extremely significant difference seen with $\mathrm{p}$ values of 0.0004 .

The table shows a comparison of mean values and SD of WOMAC scale scores in Group A and Group B. The values were compared by applying unpaired t-test. Pre-treatment shows that there is no significant difference in the WOMAC scores $(\mathrm{p}=0.5515)$, whereas post-treatment shows extremely significant difference $(p=0.0004)$ (Table 5).

\section{DISCUSSION}

Reviewing various studies, it was analyzed that the use of ultrasound, IFT, TENS, isometric exercises were the lines of treatment accompanied by exercise program for $\mathrm{OA}$ of knee joint [8].

This study was undertaken considering all the mentioned points, and the aim of this study was to evaluate the effect of circuit training in $\mathrm{OA}$ knee joint. Based on the principal of circuit training, it involves series of exercise activities. At the end of the last activity, the individual starts from the beginning and again moves through the series. The series of activity is repeated several times.

\section{Advantages of "circuit training"}

The use of circuit training can improve both strength and endurance by stressing both the aerobic and anaerobic system. Many physiological changes can occur with circuit training in various systems such as cardiovascular, respiratory, and metabolic, It also helps in decreasing body fat, decrease in blood cholesterol level, increase heat acclimatization, increase in the strength of bone and ligaments and tensile strength of tendons [11].

The study was carried out, and the result was drawn using VAS and WOMAC score as the outcome measures. A total of 30 patients ( 18 males and 12 females), out of which 17 were right and 13 were left side

Table 2: Intragroup comparison using paired t-test

\begin{tabular}{llllr}
\hline Groups & \multicolumn{2}{l}{ Mean \pm SD } & p value & Inference \\
\cline { 2 - 3 } & Pre-interventional & Post-interventional & & \\
\hline Group A & $6.22 \pm 2.1$ & $1.933 \pm 0.8837$ & $<0.0001$ & Extremely significant \\
Group B & $5.5 \pm 1.669$ & $3.8 \pm 1.612$ & $<0.0001$ & Extremely significant \\
\hline
\end{tabular}

SD: Standard deviation

Table 3: Intragroup comparison for (WOMAC) using paired t-test

\begin{tabular}{lllll}
\hline Groups & Mean \pm SD & p value & Inference \\
\cline { 2 - 3 } & Pre-interventional & Post-interventional & & \\
\hline Group A & $59.866 \pm 19.045$ & $23.266 \pm 9.169$ & $<0.0001$ & Extremely significant \\
Group B & $55.733 \pm 18.510$ & $41.933 \pm 15.586$ & 0.0110 & Statistically significant \\
\hline
\end{tabular}


Table 4: Comparison of pre- and post- VAS score between group

\begin{tabular}{lll}
\hline Groups & Mean \pm SD & \\
\cline { 2 - 3 } & Pre-interventional & Post-interventional \\
\hline Group A & $6.2 \pm 2.1$ & $1.933 \pm 0.8837$ \\
Group B & $5.5 \pm 1.669$ & $3.8 \pm 1.612$ \\
p value & 0.3075 & 0.0005 \\
Inference & Not significant & Extremely significant \\
\hline
\end{tabular}

VAS: Visual analog scale, SD: Standard deviation

Table 5: Comparison of pre- and post- WOMAC score between groups

\begin{tabular}{lll}
\hline Groups & Mean \pm SD & \\
\cline { 2 - 3 } & Pre-interventional & Post-interventional \\
\hline Group A & $59.86 \pm 19.045$ & $23.26 \pm 9.169$ \\
Group B & $55.733 \pm 18.510$ & $41.93 \pm 15.586$ \\
p value & 0.5515 & 0.0004 \\
Inference & Not significant & Extremely significant \\
\hline
\end{tabular}

WOMAC: Western Ontario and McMaster University OA index, SD: Standard deviation

affected, diagnosed as unilateral OA knee. The age group was between 50 and 60 years. Study place was Krishna College of Physiotherapy, Outpatient Department. Patients were evaluated and were divided into two Groups by convenient sampling with random allocation. Group A included 15 subjects treated with IFT and isometric exercises (static hamstrings, static quadriceps), circuit training. Group B included 15 subjects treated with IFT and isometric exercises (static hamstrings, static quadriceps).

A pre-treatment outcome measure using VAS and WOMAC scale was done [12]. The specific treatment protocol was followed as per the Group for 4 weeks, and the post-treatment outcome using VAS and WOMAC scale were documented accordingly. An exercise program was designed, and a proper ergonomic advice was given.

Intragroup comparison (within the group) was analyzed statistically using paired t-test for VAS, and WOMAC scale scores, intergroup comparison (between group) was analyzed statistically using unpaired t-test.

Intragroup comparison (within the group) was analyzed statistically using paired t-test for VAS and WOMAC scale scores. This shows that there is an extremely significant difference of Group A VAS $(p \leq 0.0001)$ and WOMAC $(\mathrm{p} \leq 0.0001)$ and Group B VAS $(\mathrm{p}<0.0001)$ and WOMAC $(\mathrm{p}=0.0110)$.

Intergroup comparison (between Groups) was analyzed statistically using unpaired t-test. This shows that pre-intervention there was no statistically significant difference seen with $\mathrm{p}$ values for VAS was 0.3075 and for WOMAC was 0.5515 . While on comparing the postinterventional values, the results between the two groups using unpaired t-test revealed that there was extremely significant difference seen with p value for VAS was 0.0005 and for WOMAC was 0.0004

In this study, an attempt was made to analyze the effect of IFT, circuit training, and exercises program in reducing pain improve strength in OA knee patients. This study was done to investigate the reduction of symptoms after the circuit training with conventional therapy in OA knee patients and its post-treatment evaluation in a standardized manner using VAS and WOMAC scale. The result shows extremely significant improvement with circuit training than conventional therapy alone.

The result of the current study shows that circuit training had extremely significant effect over conventional therapy in the management of OA knee joint both statistically and clinically.

\section{CONCLUSION}

Various conservative approaches are used in treating OA of knee joint such as ultrasound, TENS, IFT and exercises such as static hamstrings and static quadriceps. If we compare the effect of conventional treatment along with circuit training for the duration of 4 weeks, then it concludes that circuit training is found more effective in decreasing pain and improving the quality of life than using conventional therapy alone.

It is proved from this study that circuit training along with IFT and isometric exercises shows the extremely significant effect on $\mathrm{OA}$ patients than compare to IFT and static exercises and thus proving the alternate hypothesis.

\section{FURTHER SCOPE}

In the inclusion criteria patients with primary OA knee Grade I, II were selected where while taking other grades can also perform the study. Treatment time and sessions may be varied. Long-term follow-up may prove the efficacy of the treatment.

\section{REFERENCES}

1. Joshi J, Kotwal P. Essentials of Orthopaedics and Applied Physiotherapy. $2^{\text {nd }}$ ed. Mosby: Elsevier; 2011. p. 341

2. Messier SP, Loeser RF, Hoover JL, Semble EL, Wise CM. Osteoarthritis of the knee: Effects on gait, strength, and flexibility. Arch Phys Med Rehabil 1992;73(1):29-36.

3. Fisher NM, White SC, Yack HJ, Smolinski RJ, Pendergast DR. Muscle function and gait in patients with knee osteoarthritis before and after muscle rehabilitation. Disabil Rehabil 1997;19:47-55.

4. Pathwardhan SK, Bodas KS, Gundewar SS. Coping with arthritis using safer herbal option. Int J Pharm Pharm Sci 2010;2(1):1-11.

5. Silman AJ, Hegberg MC. Epidemiology of the Rheumatic Diseases. Oxford: Oxford University Press; 1993.

6. Shrinivas P, Swamy RK, Devi KP, Sailaja B. Assessment of dietary practice among osteo arthritis patients. Int $\mathrm{J}$ Pharm Pharm Sci 2014;6(6):578-81.

7. Hernández-Molina G, Reichenbach S, Zhang B, Lavalley M, Felson DT. Effect of therapeutic exercise for hip osteoarthritis pain: Results of a meta-analysis. Arthritis Rheum 2008;59(9):1221-8.

8. Facci LM, Nowotny JP, Tormem F, Trevisani VF. Effect on transcutaneous nerve stimulation and interferential therapy in patient with non-specific chronic low back pain: Randomized clinical trial. Sao Paulo Med J 2011;129(4):206-16.

9. Jarit GJ, Mohr KJ, Waller R, Glousman RE. The effects of home interferential therapy on post-operative pain, edema, and range of motion of the knee. Clin J Sport Med 2003;13(1):16-20.

10. Jagtap V, Shanmugam S. Effect of mechanical traction in osteoarthritis knee. Int J Sci Res 2014,3(10):440-3.

11. Kisner C, Colby LA. Therapeutic Exercises Foundation and Techniques. $6^{\text {th }}$ ed. Philadelphia, PA: F.A. Davis Company; 2012. p. 251-3.

12. Puett DW, Griffin MR. Published trials of nonmedicinal and noninvasive therapies for hip and knee osteoarthritis. Ann Intern Med 1994;121(2):133-40. 